\title{
SCALE-BEARING CHRYSOPHYTES FROM THE STATE OF SÃO PAULO, BRAZIL, 2: ADDITIONS TO THE FLORA
}

\author{
WUJEK, D. E. ${ }^{1}$ and BICUDO, C. E. M. ${ }^{2}$ \\ ${ }^{1}$ Department of Biology, Central Michigan University, Mt. Pleasant, MI 48859, USA \\ ${ }^{2}$ Seção de Ecologia, Instituto de Botânica, C.P. 4005, CEP 01061-970, São Paulo, SP, Brazil \\ Correspondence to: Carlos E. de M. Bicudo, Seção de Ecologia, Instituto de Botânica, C.P. 4005, \\ CEP 01061-970, São Paulo, SP, Brazil, e-mail: cbicudo@terra.com.br \\ Received July 14, 2003 - Accepted August 27, 2003 - Distributed November 30, 2004
}

(With 5 figures)

\section{INTRODUCTION}

Studies on silica-scaled chrysophytes (Chrysophyceae and Synurophyceae) using electron microscopy have resulted in an extensive list of taxa being reported from Brazil (Cronberg \& Hickel, 1985; Cronberg, 1989; Franceschini \& Couté, 1991; Wujek \& Bicudo, 1993; Franceschini et al., 1996a, b; Couté \& Franceschini, 1998; Kristiansen \& Menezes, 1998). These are similar to taxa reported from Argentina by Vigna and her co-workers (Vigna, 1979, 1981, 1984, 1986, 1988a, b, 1990, 1991, 1993; Kristiansen \& Vigna, 1994; Vigna \& Kristiansen, 1989, 1995a, b, 1996, 2002; Siver \& Vigna, 1996, 1997), from Chile by Dürrschmidt (1980, 1981, 1982a, b, 1983a, b, c), and from Colombia (Vigna \& Escobar, 1999).

The species presented here, and adding to our knowledge of their global distribution, are new records for Brazil.

\section{MATERIAL AND METHODS}

Samples were collected from two different localities in São Paulo State: Parque Estadual das Fontes do Ipiranga (hidrofitotério, Lago das Garças, and Lago das Ninféias), located in the metropolitan area of the city of São Paulo, and the Reserva Biológica de Mogi-Guaçu (Açude da Capivara e Açude do Jacaré), in the interior of the of São Paulo State, about $136 \mathrm{~km}$ in a straight line NNW from the city of São Paulo.

Samples were prepared as previously described (Wujek \& Bicudo, 1993). Some observations were made with a Philips CM10 TEM.

\section{RESULTS AND DISCUSSION}

During reexamination of previously collected phytoplankton samples and examination of several additional samples, four silica-scaled chrysophytes and one taxon of uncertain taxonomic placement not previously reported from Brazil were observed (Figs. 1-5; Table 1).

Taxonomic comments on species found:

Mallomonas multisetigera Dürrschmidt (Fig. 1)

Since the original description from Chile (Dürrschmidt, 1980), this has been shown to be a widely distributed, cosmopolitan species. The only other previous report from South America is from Argentina (Vigna \& Kristiansen, 1996).

Mallomonas pillula Harris var. valdiviana Dürrschmidt (Fig. 2)

Described originally from Chile (Dürrschmidt, 1982b), it has been recorded from a number of other locations worldwide, including Argentina (Vigna \& Kristiansen, 1996).

Mallomonas prora Dürrschmidt (Fig. 3)

This is the second report from South America. Besides the original description from Chile (Dürrschmidt, 1982a), it has previously been reported only from the Netherlands (Roijackers \& Kessels, 1986), Germany (Hickel \& Maaß, 1989), and the southern United States (Wee et al., 1993).

Chrysosphaerella coronacircumspina Wujek \& Kristiansen (Fig. 4)

Since the description from northern Michigan, USA, it has been shown to be a widely distributed species. The only other reports from South America are from Argentina (Vigna, 1988a) and Colombia (Vigna \& Escobar, 1999). 
TABLE 1

Silica-scaled chrysophytes from the state of São Paulo, Brazil (see Wujek \& Bicudo 1993 for date and physiochemical data).

\begin{tabular}{|l|l|}
\hline \multicolumn{1}{|c|}{ Taxon } & \multicolumn{1}{c|}{ Location } \\
\hline Synurophyceae & \\
\hline Mallomonas multisetigera Dürrschmidt & Hidrofitotério \\
\hline Mallomonas pillula Harris var. valdiviana Dürrschmidt & Lago das Ninféias \\
\hline Mallomonas prora Dürrschmidt & Açude da Capivara \\
\hline Chrysophyceae & \\
\hline Chrysosphaerella coronacircumspina Wujek \& Kristiansen & Lago das Ninféias \\
\hline Insertae sedis & \\
\hline Gyromitus disomatus Skuja & Lago das Garças \\
\hline
\end{tabular}

\section{Incertae sedis}

Gyromitus disomatus Skuja (Fig. 5)

Probably a free-living amoeba, it has been reported previously from two South American locations: Chile (Dürrschmidt, 1982a) and Argentina (Vigna, 1988b).

This organism has no obvious affinities with any taxonomic group (Swale \& Belcher, 1974). Nicholls (1979), using X-ray emission spectra, has demonstrated that the scales are composed of silica and are not calcified, thus this organism does not represent coccoliths.

The composition of Brazilian chrysophyte flora shows a great similarity to the flora of the neighboring countries of Argentina and Chile. Further investigations will certainly show the presence of a more diverse flora and contribute to our understanding of the biogeographical distribution of this group of organisms. Silica-scaled chrysophytes found in Brazil based on electron microscopy now comprise 69 taxa.

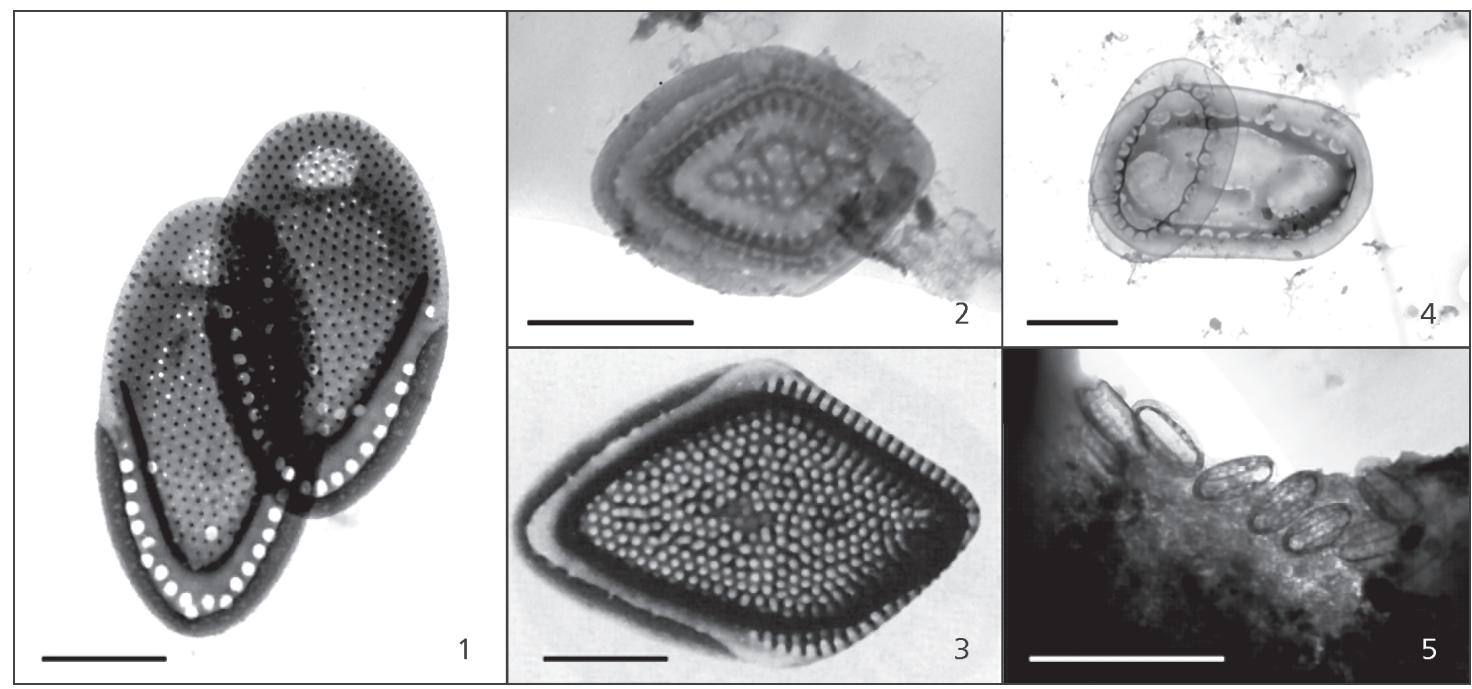

Figs. 1-3 - Mallomonas. 1. M. multisetigera Dürrschmidt. 2. M. pillula Harris var. valdiviana Dürrschmidt. 3. M. prora Dürrschmidt. Fig. 4 - Chrysosphaerella coronacircumspina Wujek \& Kristiansen. Fig. 5 - Gyromitus disomatus Skuja. Scale bar $=1 \mu \mathrm{m}$. 
Acknowledgements - D.E.W. would like to thank the Central Michigan University Research Professor Program that provided partial financial assistance for travel to Brazil, Paul Elsner for preparing coated grids, and Geoff Williams for help in plate preparation.

\section{REFERENCES}

COUTÉ, A. \& FRANCESCHINI, I. M., 1998, Scale-bearing chrysophytes from acid water of Florianopolis, Santa Catarina Island, South Brazil. Algol. Stud., 88: 37-61.

CRONBERG, G., 1989, Biogeographical studies on scaled chrysophytes from the tropics. Nova Hedwigia, 95: 191232.

CRONBERG, G. \& HICKEL, B., 1985, Mallomonas fenestrata sp. nov. and $M$. perforata sp. nov. (Chrysophyceae. Mallomonadaceae) from tropical lakes. Nord. J. Bot., 5: 105-110.

DÜRRSCHMIDT, M., 1980, Studies on the Chrysophyceae from Rio Cruces, Prov. Valdivia, South Chile, by scanning and transmission electron microscopy. Nova Hedwigia, 33: 353388.

DÜRRSCHMIDT, M., 1981, Mallomonas cristata sp. nov. (Chrysophyceae, Synuraceae) from south Chilean inland waters. Phycologia, 20: 298-302.

DÜRRSCHMIDT, M., 1982a, Studies on the Chrysophyceae from south Chilean inland waters by means of scanning and transmission electron microscopy, 2. Algol. Stud., 31: 121163.

DÜRRSCHMIDT, M., 1982b, Mallomonas parvula sp. nov. and Mallomonas retifera (Chrysophyceae, Synuraceae) from south Chile. Can. J. Bot., 60: 651-656.

DÜRRSCHMIDT, M., 1983a, Three new species of Mallomonas (Chrysophyceae, Mallomonadaceae) from Lake Lanalhue, Chile. Nord. J. Bot., 3: 423-430.

DÜRRSCHMIDT, M., 1983b, A taxonomic study of Mallomonas mangofera group (Synuraceae, Chrysophyceae) including the description of four new taxa. Pl. Syst. Evol., 143: 175196.

DÜRRSCHMIDT, M., 1983c, New taxa of the genus Mallomonas (Mallomonadaceae, Chrysophyceae) from southern Chile. Nova Hedwigia, 38: 717-726.

FRANCESCHINI, I. M. \& COUTÉ, A., 1991, Quelques Chrysophycées (Algae, Chromophyta) à écailles de l'extrême sud-est du Brésil. Algol. Stud., 62: 31-45.

FRANCESCHINI, I. M., COUTÉ, A. \& SILVA, A. Jr., 1996a, Synurophyceae et Chrysophyceae à écailles siliceuses du rio dos Sinos, RS, Brésil. Algol. Stud., 80: 59-85.

FRANCESCHINI, I. M., PRADO, J. F. \& COUTÉ, A., 1996b, Contribution à la flore des Chrysophytes à écailles siliceuses provenant de Santa Maria, RS, extrême sud du Brésil. Bull. Jard. Bot. Nat. Belg., 65: 103-116.
HICKEL, B. \& MAAß, I., 1989, Scaled chrysophytes, including heterotrophic nanoflagellates from the lake district in Holstein, northern Germany. Beih. Nova Hedwigia, 95: 233257.

KRISTIANSEN, J. \& MENEZES, M., 1998, Silica-scaled chrysophytes from an Amazonian flood-plain lake, Mussurá, northern Brazil. Algol. Stud., 90: 97-118.

KRISTIANSEN, J. \& VIGNA, M. S., 1994, Tubular scales in Synura as the possible origin of bristles in Mallomonas (Synurophyceae). Phycologia, 33: 67-70.

NICHOLLS, K. H., 1979, Is Hymenomonas prenanti Lecal (Prymnesiophyceae) really the colourless flagellate Gyromitus disomatus Skuja? Phycologia, 18: 420-423.

ROIJACKERS, R. M. M. \& KESSELS, H., 1986, Ecological characteristics of scale-bearing Chrysophyceae from the Netherlands. Nord. J. Bot., 6: 373-385.

SIVER, P. A. \& VIGNA, M. S., 1996, Notes on species of Mallomonas in the series Peronoides (Mallomonadaceae, Synurophyceae). Nord. J. Bot., 16: 223-231.

SIVER, P. A. \& VIGNA, M. S., 1997, The distribution of scaled chrysophytes in the delta region of the Paraná river, Argentina. Nova Hedwigia, 64: 421-453.

SWALE, E. M. F. \& BELCHER, J. H., 1974, Gyromitus disomatus Skuja-a free living colourless flagellate. Arch. Protistenk., 116: 211-220.

VIGNA, M. S., 1979, Ultrastructura de las escamas de Synura petersenii Korsh. var. bonaerensis nov. forma (Ochromonadales). Boln Soc. Arg. Bot., 18: 47-51.

VIGNA, M. S., 1981, Estudio con microscopio electrónico de barrido de nevos taxones de la familia Synuraceae (Chrysophyceae). Physis (B.A.), 39: 15-20.

VIGNA, M. S., 1984, Estudio de la estatospora de Mallomonas aerolata Nygaard con microscopio electrónico de barrido (Chrysophyceae). Physis (B.A.), 42: 99-101.

VIGNA, M. S., 1986, Notas algológicas XI: Dos nuevos géneros para Argentina: Mallomonas Matv. y Paraphysomonas de Saed. Darwiniana, 27: 293-304.

VIGNA, M. S., 1988a, Contribution to the knowledge of Argentine Mallomonadaceae. Nova Hedwigia, 47: 129-144.

VIGNA, M. S., 1988b, Gyromitus disomatus Skuja, flagelado incoloro: nuevo registro para Argentina. Physis (B.A.), 46: 91-92.

VIGNA, M. S., 1990, Silica-scaled, subtropical chrysophytes from the Uruguay River, Argentina. Phycologia, 29: 524540 .

VIGNA, M. S., 1991, Crisofitas escamosas (Mallomonadaceae y Paraphysomonadaceae) del Parque Nacional Nahuel-Huapi (Argentina), 1. Boln Soc. Argent. Bot., 27: 149-155.

VIGNA, M. S., 1993, Chrysophyta escamosas (Synuraceae) de una turbera de Tierra del Fuego (Argentina). Darwiniana, 32: 299-302. 
VIGNA, M. S. \& ESCOBAR, S. D., 1999, Silica-scaled chrysophytes from the Amazonian region in Colombia. Nova Hedwigia, 69: 151-162.

VIGNA, M. S. \& KRISTIANSEN, J., 1989, Mallomonas corymbosa var. interrupta nov. var. (Synuraceae) from Argentina. Nord. J. Bot., 8: 533-555.

VIGNA, M. S. \& KRISTIANSEN, J., 1995a, Mallomonas fuegiana (Synurophyceae), a new species of silica scaled chrysophytes from Tierra del Fuego, Argentina. Nord. J. Bot., 15: 439-442.

VIGNA, M. S. \& KRISTIANSEN, J., 1995b, Mallomonas heterospina and M. americana (Synuraceae) in Tierra del Fuego, Argentina, with remarks on their taxonomical interrelation. Darwiniana, 33: 61-65.
VIGNA, M. S. \& KRISTIANSEN, J., 1996, Biogeographic implications of new records of scale-bearing chrysophytes from Tierra del Fuego (Argentina). Arch. Protistenk., 147: 137-144.

VIGNA, M. S. \& KRISTIANSEN, J., 2002, Chrysophyceae y Synurophyceae de Tierra del Fuego. Monografias del Museo Argentino de Ciencias Naturales, n. 9, 45p.

WEE, J. L., BOOTH, D. J., \& BOSSIER, M. A., 1993, Synurophyceae from the southern Atlantic Coastal Plain of North America: A preliminary survey in Louisiana, USA Nord. J. Bot., 13: 95-106.

WUJEK, D. E. \& BICUDO, C. E. M., 1993, Scale-bearing chrysophytes from the state of São Paulo, Brazil. Nova Hedwigia, 56: 247-257. 